\title{
Prolonged Survival in Hereditary Surfactant Protein B (SP-B) Deficiency Associated with a Novel Splicing Mutation
}

\author{
ALSTON E. DUNBAR III, SUSAN E. WERT, MACHIKO IKEGAMI, JEFFREY A. WHITSETT, \\ AARON HAMVAS, FRANCES V. WHITE, BRUNO PIEDBOEUF, CATHERINE JOBIN, \\ SUSAN GUTTENTAG, AND LAWRENCE M. NOGEE
}

Department of Pediatrics [A.E.D., L.M.N.], Division of Neonatology, Johns Hopkins University, Baltimore, Maryland 21287, U.S.A.; Department of Pediatrics [S.E.W., M.I., J.A.W.], Divisions of Pulmonary Biology and Neonatology, Children's Hospital Medical Center, Cincinnati, Ohio 45229, U.S.A.; Department of Pediatrics [A.H.], Division of Newborn Medicine, Washington University School of Medicine and St. Louis Children's Hospital, St. Louis, Missouri 63110, U.S.A.; Department of Pathology [F.V.W.], Lauren $V$. Ackerman Laboratory of Surgical Pathology, Washington University School of Medicine, St. Louis, Missouri 63110, U.S.A.; Pediatric Unit [B.P., C.J.], Research Center, Laval University, St. Foy, Quebec, Canada G1V 4G2; and Department of Pediatrics [S.G.], Division of Neonatology, Children's Hospital of Philadelphia, Philadelphia, Pennsylvania 19104, U.S.A.

\begin{abstract}
ABST
Hereditary surfactant protein B (SP-B) deficiency has been
lethal in the first year of life without lung transplantation. We
tested the hypothesis that SP-B gene mutations may result in
milder phenotypes by investigating the mechanisms for lung
disease in two children with less severe symptoms than have
been previously observed in SP-B deficiency. Immunostaining
patterns for pulmonary surfactant proteins were consistent with
SP-B deficiency in both children. DNA sequence analysis indi-
cated that both children were homozygous for a mutation in exon
5 that created an alternative splice site. Reverse transcriptase
PCR and sequence analysis confirmed use of this splice site,
which resulted in a frameshift and a premature termination codon
in exon 7 . The predominant reverse transcriptase PCR product,
however, lacked exon 7, which restored the reading frame but
would not allow translation of the exons that encode mature
SP-B. Western blot analysis detected reduced amounts of mature
\end{abstract}
Pulmonary surfactant is a complex of lipids and proteins essential for normal lung function. SP-B facilitates spreading of surfactant lipids, formation of TM, and metabolism of surfactant components (1). The absence of SP-B in mice

Received December 29, 1999; accepted February 2, 2000

Correspondence: Lawrence Nogee, M.D., CMSC 210, 600 North Wolfe St., Baltimore, MD 21287, U.S.A.

Supported by NIH HL54703 (L.M.N.), HL54187 (L.M.N.), HL56387 (S.E.W., M.I., J.A.W.), and HL38859 (J.A.W.). A.E.D. and L.M.N. are supported by the Eudowood Foundation.
SP-B as well as an aberrant SP-B proprotein that corresponded to the size expected from translation of the abnormal transcript. We conclude that a novel splicing mutation was the cause of lung disease in these children and that hereditary SP-B deficiency can be the cause of lung disease in older children. (Pediatr Res 48: 275-282, 2000)

\begin{tabular}{|c|}
\hline $\begin{array}{l}\text { SP-B, surfactant protein B } \\
\text { proSP-B, SP-B proprotein } \\
\text { SP-C, surfactant protein C } \\
\text { proSP-C, SP-C proprotein } \\
\text { RG-PCR, restriction site generating PCR } \\
\text { LB, lamellar body } \\
\text { TM, tubular myelin } \\
\text { Sat PC, saturated phosphatidylcholine }\end{array}$ \\
\hline
\end{tabular}

genetically engineered to be homozygous for SP-B null alleles and in human infants with an inherited deficiency of SP-B results in lethal respiratory distress $(2,3)$. Hereditary SP-B deficiency is an autosomal recessive disorder that typically presents in neonates shortly after birth with unrelenting respiratory distress and a chest radiographic appearance similar to respiratory distress syndrome. The gene for SP-B has 11 exons spanning $9.5 \mathrm{~kb}$ on the short arm of chromosome $2(4,5)$. Translation of SP-B mRNA yields a preproprotein (proSP-B) that is processed to a 79-amino acid mature SP-B protein 
encoded by exons 6 and 7 (6). The most common mutation causing SP-B deficiency is an insertion in exon 4 (121ins2) that results in a frameshift and a premature codon for the termination of translation and is associated with a marked decrease in SP-B mRNA and the complete absence of proSP-B and mature SP-B proteins (3). Although a child who was a compound heterozygote for SP-B gene mutations resulting in a partial deficiency of SP-B has been reported, there has been little variation in the phenotype of SP-B deficiency (7). With aggressive respiratory support, SP-B-deficient infants have survived outside the neonatal period, but, without lung transplantation, the disease has been lethal in the first year of life in all children identified to date (8). We tested the hypothesis that mutations in the SP-B gene may result in milder lung disease by investigating the mechanisms for lung disease in two unrelated children of French-Canadian descent. The first was a full-term infant who was oxygen but not ventilator dependent at 1 mo of age, and the second was an ambulatory 6-y-old with interstitial lung disease and a lifelong oxygen requirement.

\section{METHODS}

\section{Case Histories}

Patient 1. Patient 1 was a $3.5-\mathrm{kg}$ male infant born at $40 \mathrm{wk}$ gestation via cesarean section because of fetal distress who developed respiratory distress at $8 \mathrm{~h}$ of age. Chest radiographic appearance was consistent with respiratory distress syndrome. He developed a spontaneous pneumothorax at $18 \mathrm{~h}$ of age that required thoracostomy drainage and conventional mechanical ventilation. He did not receive surfactant replacement. He was extubated but remained tachypneic and required supplemental oxygen. Dexamethasone did not improve his respiratory symptoms. There was no family history of neonatal respiratory distress, and the parents were not related. Because of persistent respiratory symptoms, he underwent an open lung biopsy and had blood drawn to evaluate for surfactant protein deficiency at 5 wk of life. While he remained extubated, his respiratory function progressively declined, and he underwent bilateral lung transplantation at 4 mo of age.

Patient 2. Patient 2 was a full-term female born after an uncomplicated pregnancy who developed respiratory distress shortly after birth but did not require mechanical ventilation. She had a persistent oxygen requirement prompting an open lung biopsy at 2 mo of age, with histopathologic changes that included interstitial fibrosis and alveolar proteinosis, although no clinical diagnosis was established. Her respiratory symptoms persisted despite diuretic and steroid therapy. She was discharged at 6 mo of life with home oxygen therapy. Her course has been complicated by the progressive development of pulmonary hypertension and right ventricular hypertrophy. A trial of cyclophosphamide was not effective in treating her lung disease. She required a brief period of mechanical ventilation at 2 y of age as a result of pneumonia. She has now been stable for several years with a persistent oxygen requirement. She is ambulatory and attends school $5 \mathrm{~d}$ a week. There was no family history of neonatal lung disease, and the parents were unrelated.
A protocol for the evaluation of infants with unexplained neonatal respiratory disease was approved by the Institutional Review Board of the respective institutions. Informed consent was obtained before obtaining blood for genetic studies.

Antisera, immunohistochemistry, and protein analysis. Antisera specific for mature SP-B (28031), full-length proSP-B (55522), the amino terminal portion of proSP-B (55019), the carboxy terminal portion of proSP-B (96189), full-length proSP-C (76983), and the amino terminal portion of proSP-C (68514) were generated in rabbits as previously described (3). Immunostaining of formalin-fixed paraffin-embedded lung biopsy specimens for surfactant proteins was performed with and without antigen retrieval using methods that have been previously described (9). A Vectastain ABC peroxidase elite rabbit IgG kit was used to detect the antigen-antibody complexes (Vector Laboratories, Inc., Burlingame, CA, U.S.A.). Lung homogenates were prepared from sections of right lower and left lower lobe from patient 1 that were obtained at the time of transplant and snap-frozen in liquid nitrogen. Homogenates were separated by SDS-PAGE, transferred to nitrocellulose paper, and immunoreacted for surfactant proteins (3). Proteins were detected by enhanced chemiluminescence using a horseradish peroxidase conjugated secondary antibody (Amersham Life Sciences, Arlington Heights, IL, U.S.A.) with exposure to Kodak X-ray film. The relative amounts of SP-B detected by Western blot analysis were quantified by densitometric analysis using a commercially available imager and software package (Gel-Doc 2000 and Quantity One, Bio-Rad, Hercules, CA, U.S.A.) under exposure conditions that provided a linear response to the amount of protein loaded.

Electron microscopy. Tissue for electron microscopy was fixed in $3 \%$ glutaraldehyde and postfixed in 1\% osmium tetroxide $\left(\mathrm{OsO}_{4}\right)$ in $0.1 \mathrm{M}$ sodium cacodylate buffer. Tissue cubes were stained with $3 \%$ uranyl acetate, dehydrated in increasing concentrations of alcohol, and embedded in spurr plastic. Thin sections were mounted on plain copper grids and stained with uranyl acetate and lead citrate.

DNA analysis. Genomic DNA was isolated from peripheral blood (Puregene, Gentra Systems, Minneapolis, MN, U.S.A.). Coding regions and flanking intron sequences of the SP-B gene were amplified by PCR as previously described (9). PCR products were sequenced using SP-B specific primers end labeled with ${ }^{33} \mathrm{P}$-dATP using $\mathrm{T}_{4}$ polynucleotide kinase (New England Biolabs, Beverly, MA, U.S.A.) and thermocycle sequencing (Thermosequenase, Amersham Life Sciences, Cleveland, OH, U.S.A.). Sequencing reaction products were separated on $6 \%$ polyacrylamide gels and visualized by autoradiography after exposure to Kodak x-ray film. Patient SP-B genomic sequences were compared with published SP-B gene sequence (4).

$\boldsymbol{R} \boldsymbol{G}-\boldsymbol{P C} \boldsymbol{R}$. An antisense primer (AGGTTTGGGCAGGGGGTCTGACGT; nucleotides 2480-2503) was designed with a single base pair difference $(\mathrm{A} \rightarrow G)$ from the wild-type sequence so that an $R s a$ I restriction site was introduced in amplimers derived from mutant alleles. This primer was used with a sense primer and conditions that have been previously described to amplify 193 bp of the SP-B gene (10). PCR products were digested with RsaI (New England Biolabs, 
Beverly, MA, U.S.A.), and the restriction fragments were separated on 3:1 Nuseive (FMC Bioproducts, Rockland, ME, U.S.A.) gels and visualized using ethidium bromide under UV light. DNA from 50 anonymous control individuals without lung disease was analyzed for the presence of the mutant allele.

$\boldsymbol{R} \boldsymbol{N A}$ analysis. RNA was prepared from lung tissue by an acid phenol extraction method (Trizol, BRL Life Technologies, Gaithersburg, MD, U.S.A.). SP-B cDNA fragments were generated using reverse transcriptase PCR (RT-PCR) and subcloned into a plasmid vector (TA cloning vector, Invitrogen, San Diego, CA, U.S.A.) as previously described (11). Automated sequencing of cDNA fragments was performed by the DNA analysis facility of the Johns Hopkins University School of Medicine, and this sequence was compared with the known SP-B cDNA sequence (12). Sequence analysis software (Gene Runner version 3.05, Hastings, NY, U.S.A.) was used to calculate the expected molecular weight of a protein produced by translation of aberrant transcripts.

Two antisense primers corresponding to sequences in exons 6 and 8 were designed to amplify transcripts lacking exon 7 (13). These primers incorporated the last eight nucleotides of exon 6 and either the first 15 nucleotides of exon 8 [GCGGCAGCCATTCTCCTTGGGAA (antisense nucleotides 2506-2513/4703-4717)] or the 13th-28th nucleotides of exon 8 [CTCCTGTCGGCGACCCTTGGGAA (antisense nucleotides 2506-2513/4715-4728)] to account for the use of a known cryptic splice site in exon 8 . These primers were used in separate PCR reactions with a common sense primer (TTCCTGGAGCAGGAGTGC, nucleotides 1471-1488) for $35 \mathrm{cy}$ cles $\left(95^{\circ} \mathrm{C} \times 20 \mathrm{~s}, 64^{\circ} \mathrm{C} \times 20 \mathrm{~s}, 72^{\circ} \mathrm{C} \times 45 \mathrm{~s}\right)$ followed by extension for $10 \mathrm{~min}$ at $72^{\circ} \mathrm{C}$. PCR products were separated on $2 \%$ agarose gels containing ethidium bromide and visualized under UV light.

Northern blot analysis of total RNA was performed by hybridization with SP-B cDNA according to methods that have been previously described (3). The cDNA probes were labeled using a random primer labeling system, and hybridized probes were detected by chemiluminescence (Gene Images, Amersham Life Sciences, Arlington Heights, IL, U.S.A.) after exposure to Kodak x-ray film.

Phospholipid analysis. Frozen lung tissue was homogenized in $0.9 \% \mathrm{NaCl}$. Aliquots of lung tissue were extracted with chloroform-methanol (2:1), and Sat PC was recovered by neutral alumina column chromatography after exposure to osmium tetroxide and was quantified by phosphorus assay (14, 15). Total protein in lung homogenates was measured with the method of Lowry et al. (16). For measurements of phospholipid composition, extracted lipid from lung tissue was used for two-dimensional thin-layer chromatography. The spots were visualized with iodine vapor, scraped, and assayed for phosphorus content (17). Explanted lung tissue from infants who were homozygous for the 121 ins 2 mutation and unused donor lung tissue were used as controls.

\section{RESULTS}

Histopathology and electron microscopy. Histologic examination of lung tissue from both patients revealed variable alveolar septal widening with increased cellularity and type II pneumocyte hyperplasia. The open lung biopsy of patient 2 and the explanted lungs of patient 1 had alveolar spaces filled with granular eosinophilic material admixed with foamy macrophages and desquamated pneumocytes. Alveolar eosinophilic material was nominal in the open lung biopsy from patient 1 . Electron microscopy of the explanted lung tissue from patient 1 (Fig. $1 A$ ) revealed numerous apically polarized cytoplasmic structures resembling LB in hyperplastic type II pneumocytes. A few of the LB-like structures had compact evenly spaced lamella; however, most contained noncompact irregularly spaced membranous whorls in contrast with the well-organized LB seen in non-SP-B-deficient lung tissue (Fig. 1B). LB-like structures were often grouped together in clusters of two or more and occasionally with multivesicular structures. Alveolar spaces contained LB-like structures, small multivesicular structures, and amorphous material. Focally, ribbon-like material coated the surface of pneumocytes, and there were rare
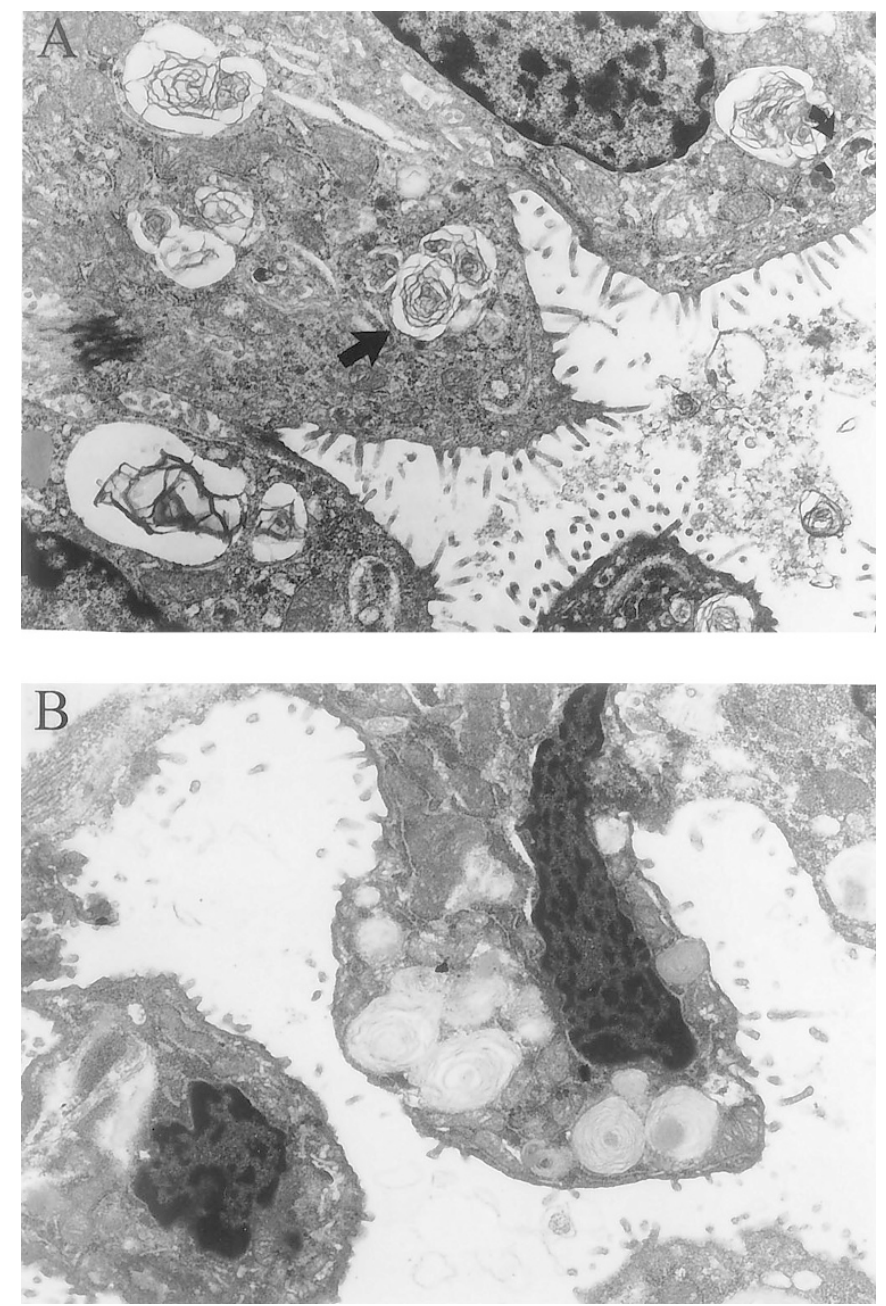

Figure 1. Electron microscopy. (A) Explanted tissue from patient 1. Hyperplastic type II pneumocytes contain numerous apically polarized LB-like structures (large arrow); multivesicular structures are also present (small arrow). The alveolar space contains LB-like structures, small multivesicular structures, and amorphous material. (B) Control tissue from a 3-mo-old child with pulmonary hypoplasia secondary to congenital diaphragmatic hernia. A type II pneumocyte contains well-organized LB, some with a dense core matrix. There is minimal alveolar material. 
haphazardly arranged structures suggestive of abnormally formed TM; however, definite TM was not identified.

Immunohistochemistry. Immunohistochemical analysis was performed to assess surfactant protein expression and distribution. SP-B staining was markedly reduced compared with controls and varied between the patients. Using a 1:2000 dilution of antisera specific for mature SP-B, staining was readily detected in control lung tissue (Fig. $2 A$ ), whereas no staining was detected in lung tissue from patient 1 (not shown), and only very weak variable staining was detected in patient 2 (Fig. 2B). Using a 1:1000 dilution of the same antisera, no staining was detected in lung tissue from patient 1 (Fig. 2C), although staining was now readily detected in tissue from patient 2 (Fig. 2D). Similarly, staining for proSP-B was not detected in patient 1 , but it was readily detected in lung tissue from patient 2 (not shown). Antigen retrieval techniques resulted in detection of both mature SP-B and proSP-B staining in patient 1 (not shown) and enhanced staining for mature SP-B and proSP-B in patient 2 (not shown). In both patients, proSP-B staining was restricted to type II pneumocytes (not shown), whereas focal staining for mature SP-B was observed in the material in the alveolar lumen as well as in type II cells. ProSP-C staining was intense in the alveolar luminal material and in type II cells from both patients (Fig. 2, $E$ and $F$ ).

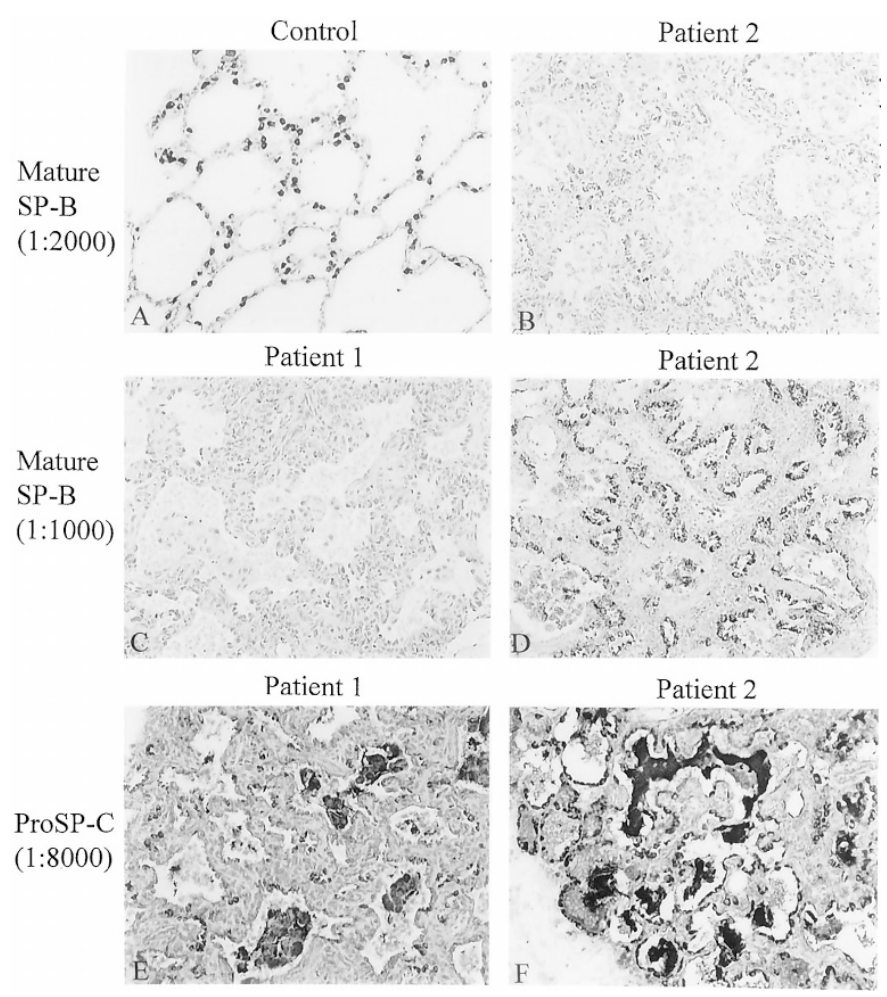

Figure 2. Immunohistochemical analysis of lung biopsy specimens for surfactant proteins. Panels $A$ and $B$ were reacted with antisera specific for mature SP-B (28031, 1:2000 dilution). (A) Control, positive for SP-B; $(B)$ Patient 2, weakly positive for SP-B. Panels $C$ and $D$ were reacted with antisera specific for mature SP-B (28031, 1:1000 dilution). (C) Patient 1, negative for SP-B; $(D)$ Patient 2, positive for SP-B. Panels $E$ and $F$ were reacted with antisera specific for proSP-C (68514). ProSP-C staining was intense in the alveolar luminal material and in type II cells from both patients. All panels are shown at $\times 230$ magnification.
DNA analysis. A $\mathrm{G} \rightarrow \mathrm{T}$ transversion at genomic nucleotide 2479 (cDNA nucleotide 479) was identified in both patients and was the only deviation from the published SP-B coding sequences. This substitution is in the wobble position of codon 155 in exon 5 and would not be predicted to alter the amino acid sequence, although it would create an alternate donor splice site (AGGGATGT $\rightarrow$ AGGTATGT). Utilization of this splice site would delete 119 bases of exon 5 from the mRNA and result in a frameshift with the introduction of a premature termination codon in exon 7.

RG-PCR was used to screen genomic DNA for c.479G $>$ T alleles. Amplimers from both patients were completely digested on restriction analysis, suggesting that these patients were homozygous for c.479G $>\mathrm{T}$ alleles (Fig. 3). The c. $479 \mathrm{G}>\mathrm{T}$ allele was not present on restriction analysis of 100 chromosomes from control individuals without lung disease.

$\boldsymbol{R N A}$ analysis. SP-B mRNA was markedly reduced on northern analysis of RNA from patient 1 (not shown). RT-PCR was used to determine whether low levels of SP-B mRNA were present and whether such transcripts were appropriately spliced. Three different SP-B cDNA were amplified and sequenced (Fig. 4). Transcripts corresponding to both the normal SP-B mRNA sequence and those that used the newly created splice site and had 119 bases of exon 5 sequence deleted were identified. However, the major RT-PCR product was approximately 300 bases smaller than that generated from control lung mRNA. Sequence analysis demonstrated that this cDNA resulted from use of the novel splice site and lacked 119 bases of exon 5 sequence as well as the entire sequence from exon 7 (184 bp). This deletion eliminated the premature termination codon and restored the reading frame.

To discover whether skipping of exon 7 was selective for this mutation, RT-PCR was used to amplify transcripts lacking exon 7. Two different antisense primers were constructed to amplify transcripts using the normal exon 8 acceptor splice site as well as those using a known cryptic splice site excluding the first 12 bases of exon $8(11,18)$. Transcripts lacking exon 7 were identified in RNA from normal tissue (Fig. 5). When these primers were used with a different sense primer and genomic DNA as a template to amplify a fragment of compa-

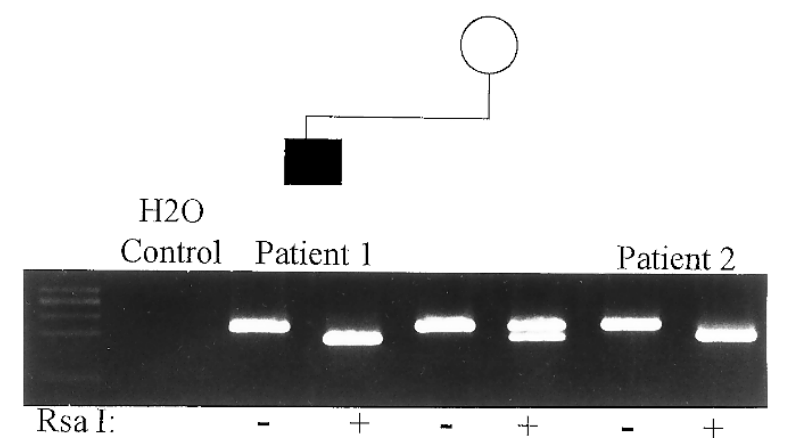

Figure 3. RG-PCR and restriction analysis. RsaI digestion of 193 bp amplimers from c.479G $>$ T alleles yields fragments of 169 and $24 \mathrm{bp}$, whereas amplimers from wild-type alleles are not digested. Amplimers from patient 1 and 2 were completely digested, consistent with these children being homozygous for the c.479G $>\mathrm{T}$ mutation. After RsaI digestion of amplimers from patient 1 's mother, the presence of bands at both 193 and $169 \mathrm{bp}$ indicates that she had the c.479G $>$ T mutation on one allele. 

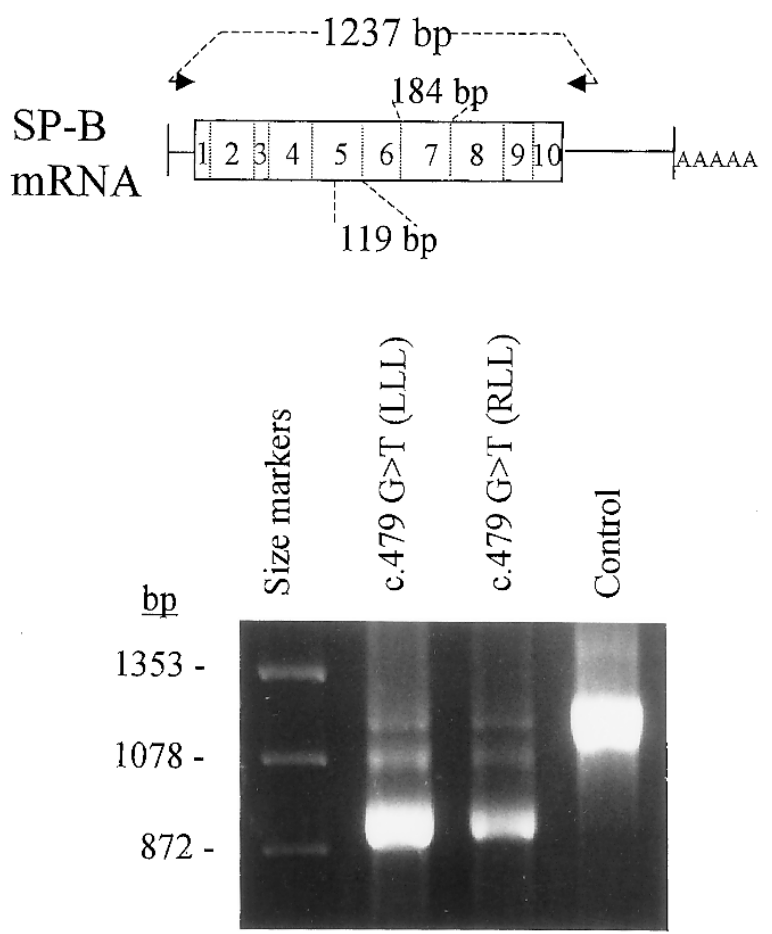

Figure 4. RT-PCR amplification of SP-B transcripts. An RT-PCR product of the predicted size (1237 bp) was observed in control tissue (lane 4). The predominant product in tissues from patient 1 (lanes 2 and 3) was approximately $300 \mathrm{bp}$ shorter, corresponding to the size predicted from the deletions of part of exon 5 and all of exon 7. Less intense products were also observed in the patient samples, corresponding to the sizes of the normal transcript and those with a deletion of part of exon 5 .

rable length, no products were observed, indicating that the 8 bp of these primers corresponding to exon 6 were not sufficient for PCR amplification under the same conditions (not shown).

Protein analysis. ProSP-B of $M_{\mathrm{r}}=40,000 \mathrm{D}$ and a processing intermediate of $M_{\mathrm{r}}=26,000 \mathrm{D}$ were readily detected in control lung tissues using antisera directed against proSP-B but were not observed in lung tissue from patient 1. However, these antisera detected an approximately 30,000 -D protein that was present in an amount roughly comparable to proSP-B in control tissues (Fig. 6, upper panel). The size of this protein corresponded to the size predicted from translation of transcripts with deletion of part of exon 5 and all of exon 7. Similar results were obtained with antisera specific to the entire proSP-B protein and the amino terminal regions of proSP-B (not shown). Mature SP-B was detected in homogenates of lung tissue from patient 1 (Fig. 6, lower panel); the amount of mature SP-B detected was variable but was generally reduced compared with controls. By densitometric analysis, the amount of SP-B was approximately 12 to $16 \%$ of that observed in normal lung tissue. Aberrant SP-C peptides were abundant in all lung homogenate samples from patient 1 (Fig. 6, lower panel).

Phospholipid analysis. Surfactant phospholipid contents and compositions were examined in lung tissues from patient 1 , two infants homozygous for 121 ins 2 mutation, and normal lung (Table 1). All samples were run in triplicate with similar results. The Sat PC to protein ratio was markedly increased in lung tissue from patient 1 when compared with control lung
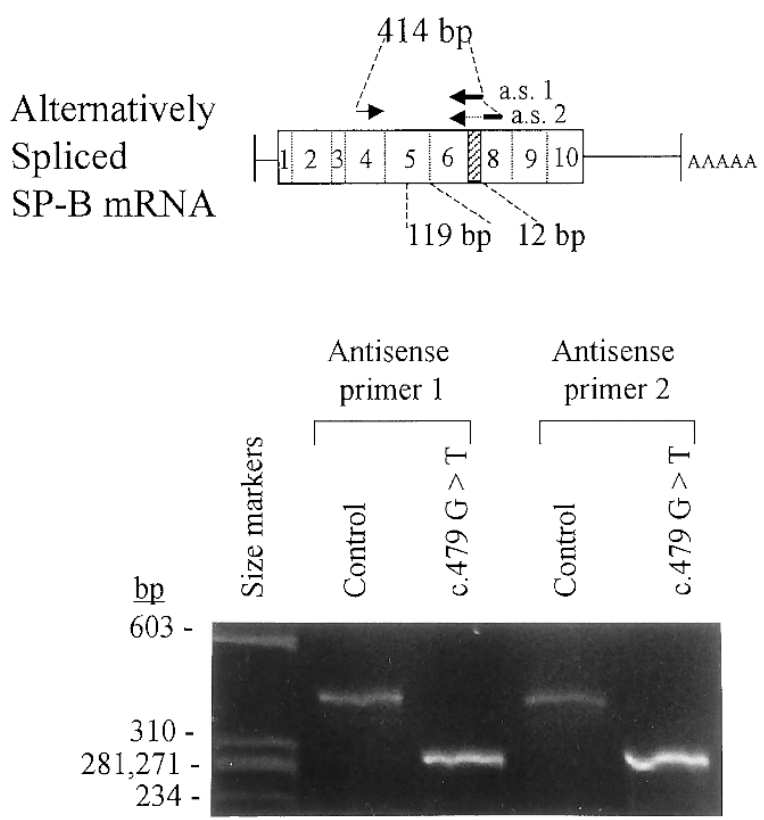

Figure 5. RT-PCR using primers specific for transcripts lacking exon 7 . RT-PCR was performed using antisense primers corresponding to part of exon 6 and either the beginning of SP-B exon 8 (antisense primer 1/a.s. 1) or the 13th-28th nucleotides of exon 8 (antisense primer 2/a.s. 2), accounting for the use of a cryptic splice site (shaded area) in exon 8. RT-PCR products of the predicted size (414 bp) were observed in control tissues (lanes 2 and 4), indicating the presence of transcripts lacking exon 7. RT-PCR products amplified from RNA isolated from patient 1 (lanes 3 and 5) were shorter by the amount predicted to result from use of the new splice site in exon 5 .

tissue, from patients with the 121 ins 2 mutation, and previously reported values (19). Phosphatidylglycerol content in lung tissue from patient 1 was lower than in control tissue, although the reduction was not as dramatic as that seen in infants homozygous for the 121 ins2 mutation (7).

\section{DISCUSSION}

Hereditary SP-B deficiency has been uniformly lethal in infancy without lung transplantation, with the majority of the infants dying in the first month of life (20). Partial SP-B deficiency has been reported in a child with chronic lung disease who was a compound heterozygote for two different SP-B gene mutations (121ins2/R236C) (7). This child, however, remained hospitalized his entire life, was ventilator dependent, and died at 9 mo of age while awaiting lung transplantation. In the present study, we report two SP-B-deficient children with less severe clinical symptoms than have been previously observed, including an ambulatory 6 -y-old who has survived into childhood without the need for ventilatory assistance or lung transplantation. Both children shared a novel splicing mutation in their SP-B genes.

Approximately $15 \%$ of the point mutations that have been identified as responsible for human genetic disease result in defective RNA splicing (21). The c.479G $>$ T mutation creates a donor splice site with an almost identical consensus value score as the donor splice site normally used for exon 5 (89.5 versus 89.3 ), suggesting that they should be equally attractive targets for splicing based on splice site sequence (22). Muta- 


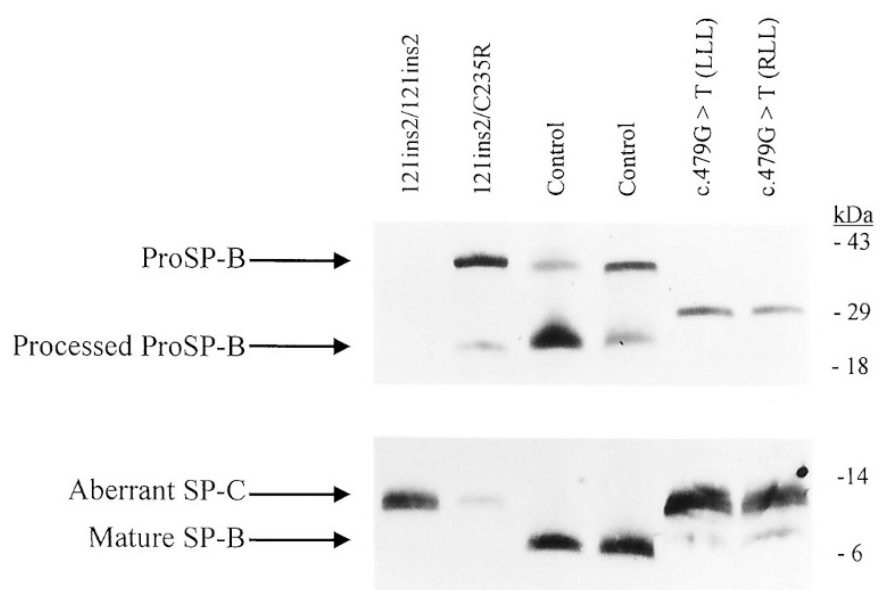

Figure 6. Western blot analysis of lung homogenates. Migration of molecular weight standards are indicated on the right. Upper panel, Western blot analysis using antisera directed against the carboxy terminal region of proSP-B. Proteins of $M_{\mathrm{r}}=40$ and $26 \mathrm{kD}$, corresponding to proSP-B and a processing intermediate, were present in control tissues (lanes 3 and 4 ) but not in tissues from an infant homozygous for the 121ins2 mutation (lane 1) or from patient 1 who was homozygous for the c. $479 \mathrm{G}>\mathrm{T}$ mutation (lanes 5 and 6 ). A $30-\mathrm{kD}$ protein was observed only in lung tissue from the infant homozygous for the c. $479 \mathrm{G}>\mathrm{T}$ mutation (lanes 5 and 6 ). Lower panel, Analysis using antisera directed against proSP-C and mature SP-B. Mature SP-B and abundant aberrant proSP-C peptides were both detected in lung tissue from patient 1 (lanes 5 and 6 ). Aberrant proSP-C peptides but not mature SP-B were detected in lung from an infant homozygous for the 121 ins 2 mutation (lane 1) and in lung tissue from an infant who was a compound heterozygote for SP-B gene mutations (lane 2). Mature SP-B but not aberrant proSP-C peptides were detected in control lung tissue (lanes 3 and 4).

Table 1. Surfactant phospholipid content and composition

\begin{tabular}{lcccc}
\hline & 121ins2 & 121ins2 & c.479G $>$ T & Normal \\
\hline Sat PC ( $\mu$ mol/mg protein) & 164 & 66 & 845 & 31 \\
Surfactant composition & & & & \\
$\quad$ (\% phospholipid) & & & & \\
Phosphatidylcholine & 63.3 & 57.6 & 69.6 & 54.1 \\
Phosphatidylglycerol & 2.6 & 2.6 & 7.7 & 16.0 \\
Phosphatidylinositol & 5.2 & 5.4 & 4.6 & 3.0 \\
Phosphatidylethanolamine & 7.5 & 14.2 & 3.5 & 9.4 \\
Phosphatidylserine & 1.8 & 2.3 & 1.0 & 3.0 \\
Lysophosphatidylcholine & 8.0 & 8.3 & 2.7 & 11.3 \\
Sphingomyelin & 3.7 & 1.2 & 3.6 & 1.6 \\
Lyso-bis-phoshatidic acid & 4.1 & 1.9 & 4.2 & 4.7 \\
Unknown & 7.0 & 6.6 & 3.1 & - \\
\hline
\end{tabular}

tions that alter splice sites have resulted in moderate phenotypes or variable penetrance through the preferential use of splice sites, a phenomenon that has been observed with several human genetic diseases including cystic fibrosis, leukocyte adhesion deficiency, and Sandhoff disease (23-25). Variable use of the normal splice site may explain the decreased severity of lung disease in these children. The presence of normally spliced transcript in RNA from patient 1 and the detection of low levels of SP-B in both lung tissue and alveolar luminal material from these children support this hypothesis. The severity of disease associated with this mutation was variable, as one child progressed to lung transplantation in infancy whereas the other has survived with medical management alone. The milder phenotype in the older child correlated with more readily detectable mature SP-B staining on immunohis- tochemical analysis, an observation that could result from different frequencies of splice site use between these patients. Alternatively, factors other than genotype such as gender or differential expression of other genes impacting on pulmonary homeostasis may have influenced the phenotypes in these children.

Although the complete absence of SP-B in both mice and humans is lethal, the level of SP-B expression required for normal pulmonary function is not clear $(2,3)$. Mice genetically engineered to be heterozygous for an SP-B null allele do not have obvious respiratory symptoms and survive to adulthood (2). However, these mice have a 50\% reduction in SP-B in lung tissue that is associated with changes in pulmonary mechanics, decreased compliance and mild air trapping, and an increased susceptibility to hyperoxic lung injury $(17,26,27)$. Pulmonary function testing in nine human adult carriers for the 121 ins 2 mutation was normal, although it is not known whether this genotype increases susceptibility to pulmonary injury in humans (28). Reduction of SP-B levels to an estimated $6 \%$ of the adult level in one infant resulted in severe newborn respiratory distress and was lethal in the first year of life despite aggressive respiratory support (7). Lung homogenates from patient 1 contained approximately 12 to $16 \%$ of adult SP-B levels; however, this child still had a pulmonary pathology and abnormal SP-C metabolism characteristically seen with SP-B deficiency. Although this child did not require aggressive ventilatory support, he did require moderate supplemental oxygen that in combination with decreased SP-B levels may have incited additional pulmonary injury. Although frozen lung tissue was not available from patient 2 to directly assess SP-B levels, immunohistochemical staining of this child's lung tissue was more intense than that seen in tissue from patient 1 when the same dilution of SP-B-specific antisera was used. We speculate that the level of SP-B production in this child more closely approximated the level required for adequate pulmonary function, allowing her survival into childhood.

Use of the alternative splice site would result in a frameshift and the introduction of a premature termination codon in exon 7; however, the majority of the transcripts also lacked exon 7. Skipping of exon 7 has been observed in infants homozygous for the 121 ins 2 mutation but has not been reported in normal lung tissue or in tissues from patients with other lung diseases (11). Premature termination codons can induce skipping of the exons in which they are contained (29-31). In this study, transcripts lacking exon 7 were demonstrated in RNA from control tissues, indicating that skipping of this exon is not the consequence of the mutation. The skipping of exon 7 in transcripts using the novel splice site would restore the reading frame, and an aberrant proSP-B protein corresponding to the size predicted from this aberrant transcript was identified, indicating that these transcripts were translated (Fig. 7).

ProSP-B has structural homology to prosaposin and includes three tandemly repeated saposin motifs, each consisting of a characteristic combination of cysteine residues paired as disulfides and accompanying hydrophobic regions (32). The saposins are glycoproteins that activate lysosomal hydrolases and are derived from proteolytic cleavage of prosaposin, a common precursor protein (33). Mature SP-B corresponds to the middle 


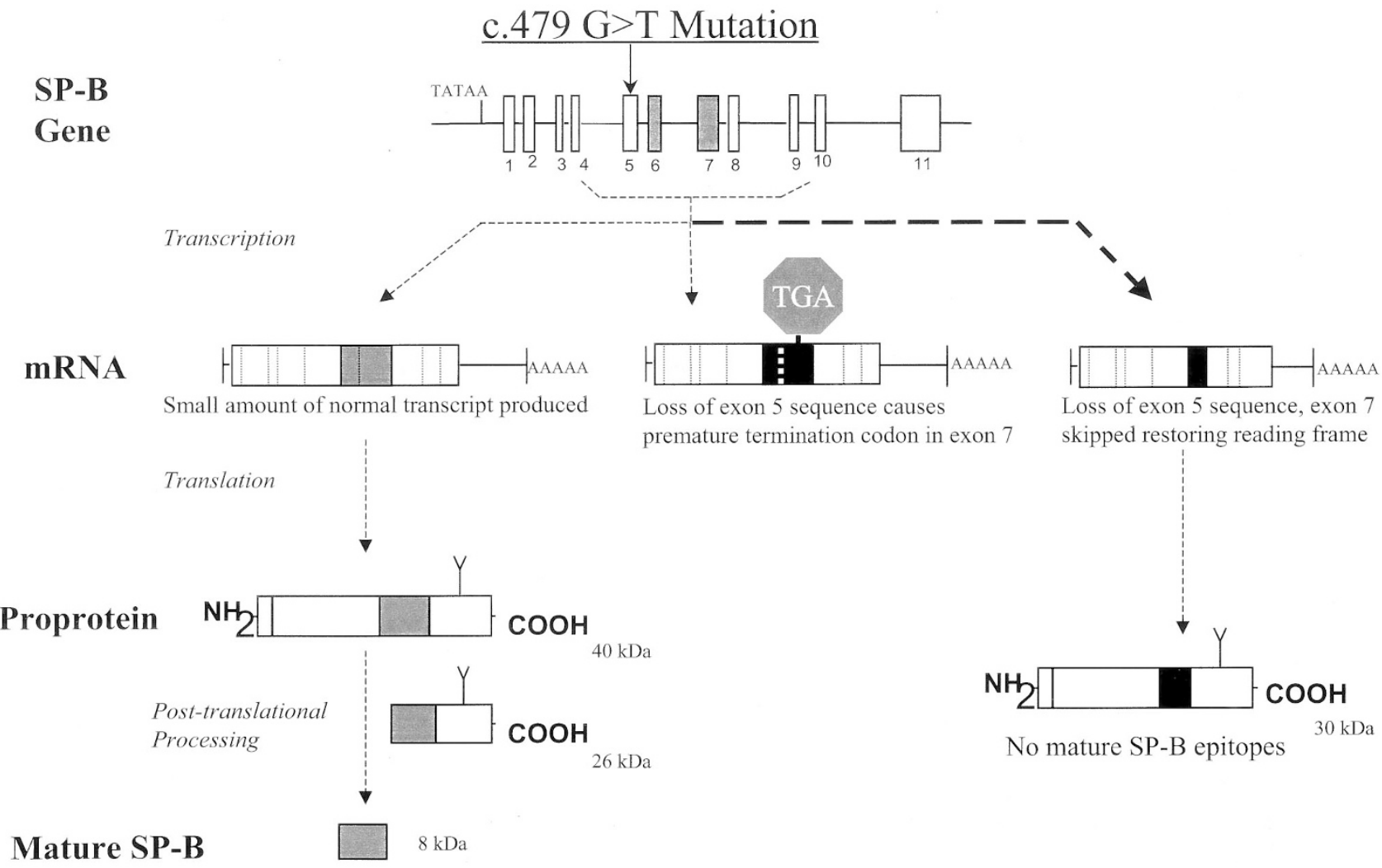

Figure 7. SP-B mRNA and protein production from c. $479 \mathrm{G}>\mathrm{T}$ alleles. Utilization of the normal exon 5 donor splice site allows for production of normal SP-B transcripts and mature SP-B protein. Use of the splice site created by the c.479G $>$ T mutation deletes 119 bases of exon 5, produces a frameshift, and introduces a premature termination codon in exon 7. However, transcripts that are also lacking exon 7, resulting in the loss of the premature termination codon, have a restored reading frame and may lead to the production of an aberrant SP-B propeptide. The proSP-B amino and carboxy terminal regions are in frame in these transcripts, and these epitopes remain immunoreactive in the aberrant protein.

saposin domain. The aberrant proSP-B protein derived from the alternatively spliced transcripts caused by the c.479G $>$ T mutation would maintain the amino and carboxy terminal saposin domains connected by a 30 -amino acid peptide that is unrelated to mature SP-B due to the frameshift and absence of exon 7. In vitro expression of SP-B constructs containing the proSP-B amino terminal domain has suggested the importance of this region for proper trafficking of the proprotein to distal cellular compartments $(34,35)$. Genetically engineered mice that expressed only SP-B constructs that lacked sequences encoding the proSP-B carboxy terminal domains produced mature SP-B but had perturbed proSP-C processing and abnormally large $\mathrm{LB}$, suggesting a role for the carboxy terminal region in LB biosynthesis and SP-C proprotein processing (36). However, SP-C metabolism was abnormal in the children we describe despite the presence of the aberrant protein, suggesting that the carboxy terminal region of the abnormal peptide was nonfunctional or that the reduced level of mature SP-B protein was also important in altered proSP-C processing. The aberrant proSP-B protein was present in amounts comparable to proSP-B in controls, even though total SP-B mRNA was reduced compared with controls, suggesting that the abnormal protein may not have been metabolized normally. Electron microscopy revealed that the aberrant protein did permit formation of LB-like structures, in contrast with that seen with the 121 ins 2 mutation that results in markedly decreased numbers of cytoplasmic LB and loss of apical polar- ization of surfactant structures (37). The phosphatidylglycerol content in lung tissue from patient 1 was intermediate between homozygous 121 ins 2 and normal levels, consistent with a partial defect in SP-B function; however, the mechanisms for the increased Sat PC content in this tissue are not clear and may be related to the presence of the abnormal protein $(7,38)$. It is possible that the milder lung disease in these children may have been related to some preserved function of the abnormal proprotein in these children, although severe lung disease has been observed in children whose mutations allowed for proSP-B production (9).

We identified a novel SP-B gene-splicing mutation as the cause of lung disease in two unrelated children of FrenchCanadian descent. Given the association with decreased and possibly variable severity of lung disease, the contribution to morbidity and mortality attributed to this mutation needs to be examined in this population. The mechanism for decreased severity of lung disease in these children remains unclear but may be related to the production of low levels of normal SP-B transcript or partial function of an aberrant proSP-B protein. We demonstrate that hereditary SP-B deficiency need not be fatal in infancy and can be the cause of lung disease in older children. These findings suggest that SP-B deficiency may be an etiology for a broader range of lung disease than previously suspected and that abnormal SP-B expression should be considered as a potential etiology in older individuals with interstitial lung disease. 
Acknowledgments. The authors thank Monica Castro, Justin Huang, and Sherri Profitt for technical assistance. Control genomic DNA was provided by Drs. Garry Cutting and Harry Deitz. Antibody to proSP-B was supplied by Dr. Tim Weaver.

\section{REFERENCES}

1. Whitsett JA, Nogee LM, Weaver TE, Horowitz AD 1995 Human surfactant protein B: structure, function, regulation, and genetic disease. Physiol Rev 75:749-757

2. Clark JC, Wert SE, Bachurski CJ, Stahlman MT, Stripp BR, Weaver TE, Whitsett JA 1995 Targeted disruption of the surfactant protein B gene disrupts homeostasis, causing respiratory failure in newborn mice. Proc Natl Acad Sci USA 92:7794-7798

3. Nogee LM, deMello DE, Dehner LP, Colten HR 1993 Brief report: deficiency of pulmonary surfactant protein $\mathrm{B}$ in congenital alveolar proteinosis. $\mathrm{N}$ Engl J Med 328:406-410

4. Pilot-Matias TJ, Kister SE, Fox JL, Kropp K, Glasser SW, Whitsett JA 1989 Structure and organization of the gene encoding human pulmonary surfactant proteolipid SP-B. DNA 8:75-86

5. Moore KJ, D'Amore-Bruno MA, Korfhagen TR, Glasser SW, Whitsett JA, Jenkins NA, Copeland NG 1992 Chromosomal localization of three pulmonary surfactant protein genes in the mouse. Genomics 12:388-393

6. O'Reilly MA, Weaver TE, Pilot-Matias TJ, Sarin VK, Gazdar AF, Whitsett JA 1989 In vitro translation, post-translational processing, and secretion of pulmonary surfactant protein B precursors. Biochim Biophys Acta 1011:140-148

7. Ballard PL, Nogee LM, Beers MF, Ballard RA, Planer BC, Polk L, deMello DE, Moxley MA, Longmore WJ 1995 Partial deficiency of surfactant protein B in an infant with chronic lung disease. Pediatrics 96:1046-1052

8. Nogee LM 1997 Surfactant protein-B deficiency. Chest 111:129S-135S

9. Nogee LM, Wert SE, Proffit SA, Hull WM, Whitsett JA 2000 Allelic heterogeneity in surfactant protein B (SP-B) deficiency. Am J Resp Crit Care Med 161:973-981

10. Klein JM, Thompson MW, Snyder JM, George TN, Whitsett JA, Bell EF, McCray PB, Nogee LM 1998 Transient surfactant protein B deficiency in a term infant with severe respiratory failure. J Pediatr 132:244-248

11. Nogee LM, Garnier G, Deitz H, Singer L, Murphy AM, deMello DE, Colten HR 1994 A mutation in the surfactant protein B gene responsible for fatal neonatal respiratory disease in multiple kindreds. J Clin Invest 93:1860-1863

12. Jacobs KA, Phelps DS, Steinbrink R, Fisch J, Kriz R, Mitsock L, Dougherty JP, Taeusch HW, Floros J 1987 Isolation of a cDNA clone encoding a high molecular weight precursor to a $6-\mathrm{kDa}$ pulmonary surfactant-associated protein. J Biol Chem 262:9808-9811

13. Dietz HC, Kendzior RJ 1994 Maintenance of an open reading frame as an additional level of scrutiny during splice site selection. Nat Genet 8:183-188

14. Mason RJ, Nellenbogen J, Clements JA 1976 Isolation of disaturated phosphatidylcholine with osmium tetroxide. J Lipid Res 17:281-284

15. Bartlett GR 1959 Phosphorous assay in column chromatography. J Biol Chem 234:466-468

16. Lowry OH, Rosebrough NJ, Farr AL, Randall RJ 1951 Protein measurement with the Folin phenol reagent. J Bio Chem 193:265-275

17. Clark JC, Weaver TE, Iwamoto HS, Ikegami M, Jobe AH, Hull WM, Whitsett JA 1997 Decreased lung compliance and air trapping in heterozygous SP-B deficient mice. Am J Respir Cell Mol Biol 16:46-52

18. Lin Z, Wang G, de Mello DE, Floros J 1999 An alternatively spliced surfactant protein B mRNA in normal lung: disease implication. Biochem J 343:145-149

19. Rebello CM, Jobe AH, Eisele JW, Ikegami M 1996 Alveolar and tissue surfactant pool sizes in humans. Am J Respir Crit Care Med 154:625-628
20. Hamvas A, Nogee LM, deMello DE, Cole FS 1995 Pathophysiology and treatment of surfactant protein-B deficiency. Biol Neonate 67:18S-31S

21. Krawczak M, Reiss J, Cooper DN 1992 The mutational spectrum of single base-pair substitutions in mRNA splice junctions of human genes: causes and consequences. Hum Genet 90:41-54

22. Shapiro MB, Senapathy P 1987 RNA splice junctions of different classes of eukaryotes: sequence statistics and functional implications in gene expression. Nucleic Acids Res 15:7155-7174

23. Chiba-Falek O, Kerem E, Shoshani T, Aviram M, Augarten A, Bentur L, Tal A, Tullis E, Rahat A, Kerem B 1998 The molecular basis of disease variability among cystic fibrosis patients carrying the $3849+10 \mathrm{~kb} \mathrm{C} \rightarrow \mathrm{T}$ mutation. Genomics 53:276-283

24. Kishimoto TK, O’Conner K, Springer TA 1989 Leukocyte adhesion deficiency. Aberrant splicing of a conserved integrin sequence causes a moderate deficiency phenotype. J Biol Chem 264:3588-3595

25. McInnes B, Potier M, Wakamatsu N, Melancon SB, Klavins MH, Tsuji S, Mahuran DJ 1992 An unusual splicing mutation in the HEXB gene is associated with dramatically different phenotypes in patients from different racial backgrounds. J Clin Invest 90:306-314

26. Tokieda K, Iwamoto HS, Bachurski C, Wert SE, Hull WM, Ikeda K, Whitsett JA 1999 Surfactant protein-B-deficient mice are susceptible to hyperoxic lung injury. Am J Respir Cell Mol Biol 21:463-472

27. Tokieda K, Whitsett JA, Clark JC, Weaver TE, Ikeda K, McConnell KB, Jobe AH, Ikegami M, Iwamoto HS 1997 Pulmonary dysfunction in neonatal SP-B-deficient mice. Am J Physiol 273:L875-L882

28. Yusen RD, Cohen AH, Hamvas A 1999 Normal lung function in subjects heterozygous for surfactant protein-B deficiency. Am J Respir Crit Care Med 159:411-414

29. Teraoka SN, Telater M, Becker-Catania S, Liang T, Önengüt S, Tolun A, Chessa L, Sanal Ö, Bernatowska E, Gatti RA, Concannon P 1999 Splicing defects in the ataxia-telangiectasia gene, ATM: underlying mutations and consequences. Am J Hum Genet 64:1617-1631

30. Dietz HC, Valle D, Francomano CA, Kendzior RJ, Pyeritz RE, Cutting GR 1993 The skipping of constitutive exons in vivo induced by nonsense mutations. Science 1993 259:680-683

31. Hull J, Shackleton S, Harris A 1994 The stop mutation R553X in the CFTR gene results in exon skipping. Genomics 19:362-364

32. Patthy L 1991 Homology of the precursor of pulmonary surfactant-associated protein SP-B with prosaposin and sulfated glycoprotein 1. J Biol Chem 266:6035-6037

33. KishimotoY, Hiraiwa M, O’Brien JS 1992 Saposins: structure, function, distribution, and molecular genetics. J Lipid Res 33:1255-1267

34. Lin S, Akinbi HT, Breslin JS, Weaver TE 1996 Structural requirements for targeting of surfactant protein B (SP-B) to secretory granules in vitro and in vivo. J Biol Chem 271:19689-19695

35. Lin S, Phillips KS, Wilder MR, Weaver TE 1996 Structural requirements for intracellular transport of pulmonary surfactant protein B (SP-B). Biochim Biophys Acta 1312:177-185

36. Akinbi HT, Breslin JS, Ikegami M, Iwamoto HS, Clark JC, Whitsett JA, Jobe AH, Weaver TE 1997 Rescue of SP-B knockout mice with a truncated SP-B proprotein. Function of the C-terminal propeptide. J Biol Chem 272:9640-9647

37. deMello DE, Heyman S, Phelps DS, Hamvas A, Nogee LM, Cole S, Colten HR 1994 Ultrastructure of lung in surfactant protein B deficiency. Am J Respir Cell Mol Biol 11:230-239

38. Beers MF, Hamvas A, Moxley MA, Gonzales LW, Guttentag SH, Solarin KO, Longmore WJ, Nogee LM, Ballard PL 2000 Pulmonary surfactant metabolism in infants lacking surfactant protein B. Am J Respir Cell Mol Biol 22:380-391 